\title{
Preface to ECDM 2008
}

\author{
Fabio Grandi \\ University of Bologna, Italy
}

Change is a fundamental but sometimes neglected aspect of information and database systems. The management of evolution and change and the ability for database, information and knowledge-based systems to deal with change is an essential component in developing and maintaining truly useful systems. Many approaches to handling evolution and change have been proposed in various areas of data management and this forum seeks to bring together researchers and practitioners from both more established areas and those from emerging areas to look at this issue. The fifth ECDM workshop (the first four ECDM workshops were held with ER'99 in Paris, France, with ER'02 in Tampere, Finland, with ER'04 in Shanghai, China, and with ER'06 in Tucson, Arizona) deals with the manner in which change can be handled, and the semantics of evolving data and data structure in computer based systems, including databases, data warehouses and Web-based information systems.

With respect to the main ER conference, the ECDM workshop series aim at stressing the evolutionary aspects involved in conceptual modelling and in development and implementation of systems, ranging from the modelling of information dynamics to the dynamics of the modelling process itself. Another explicit aim of ECDM workshops is to bring together scientists and practitioners interested in evolution and change aspects in different research fields and, thus, often belonging to completely separate communities. As a result, such an interaction could become tighter and cross-fertilization more useful in the context of a collaborative workshop like ECDM.

Following the acceptance of the workshop proposal by the ER 2008 organizing committee, an international and highly qualified program committee was assembled from research centers worldwide. As a result of the call for papers, the program committee received 8 submissions from 6 countries and after rigorous refereeing 3 high quality papers were eventually chosen for presentation at the workshop and publication in these proceedings, with a $37.5 \%$ acceptance ratio. Furthermore, the workshop program is enriched by a keynote speech given by Carlo Zaniolo, leading specialist in the temporal XML field but also enduring friend of the ECDM workshop series.

I would like to express my thanks to the program committee members and the additional external referees for their timely expertise in reviewing, the authors for submitting their papers, the ER 2008 organizers for their support. 